decline from $23.9 \%$ in 1998 to $21.8 \%$ in 2009, mortality rates deserve our worries.

Conclusions Overall, the results point out to a declining co-infection trend. However treatment outcomes are quite worrisome; the cure of co-infected patients, being lower than the non co-infected, demonstrate the need to create priorities and adequate strategies for this population, mainly regarding the follow-up of these cases.

\section{P1-280 ANALYSIS OF TUBERCULOSIS RESISTANCE IN SAO PAULO STATE (SPS), BRAZIL FROM 2007 TO 2008}

doi:10.1136/jech.2011.142976e.72

M J P Rujula,* V M N Galesi, A Guarnier, N Goldgrub, S Fukasava. Tuberculosis Division, Sao Paulo State Secretary of Health, Sao Paulo, Brazil

Introduction Tuberculosis (TB) remains a serious public health problem. The most serious aspect of this problem has been the appearance of $\mathrm{TB}$ drug-resistance (TBDR), mainly the multidrugresistance (TBMDR). One in five TB cases in Brazil occurs in Sao Paulo State, which has about 19000 cases per year. Since 1980, TB treatment in Brazil has been with rifampin, isoniazid and pirazinamid. Thus, TB-DR monitoring is essential to assess its trend, in order to better assist the TB Control Program (TCP).

Objective To analyse TBDR in Sao Paulo State during the 2007-2008 period.

Methodology Using 'population proportionate cluster sampling' we selected 30 randomised clusters to design the resistance survey. During the 2007-2008 period, all respiratory symptomatic patients of these clusters answered the questionnaire and were submitted to AFB culture and sensitivity tests (ST).

Results From the 1746 patients interviewed: $30.3 \%$ were female and $69.7 \%$ male, the mean age was 37 years, $20.0 \%$ of the patients were HIV-positive and 821 were submitted to culture and ST. The primary resistance to isoniazid and rifampin was $5.8 \%$ and $1.5 \%$, respectively. MDRTB was $1.1 \%$ and any-resistance was $9.8 \%$. The acquired-resistance was as follows: isoniazid, 20.4\%; rifampin $12.2 \%$; MDRTB $12.2 \%$ and any-resistance $22.4 \%$.

Conclusions Data on TB-DR are essential to the TCP's improvement. Results of isoniazid (increase from $4.0 \%$ to $5.8 \%$ in 8 years) showed the necessity of addition of ethambutol to our treatment schedule.

\section{P1-281 ANTIOXIDANTS FOR PREVENTING PREECLAMPSIA: A SYSTEMATIC REVIEW}

doi:10.1136/jech.2011.142976e.73

${ }^{1} \mathrm{~A}$ Magalhães, ${ }^{2} \mathrm{~L}$ Motta, ${ }^{2} \mathrm{~T}$ Galvao, ${ }^{2} \mathrm{M}$ Pereira. ${ }^{*}$ Health Department, Brasilia, Distrito Federal, Brazil; ${ }^{2}$ University of Brasilia, Brasilia, Distrito Federal, Brazil

Objective To investigate the effectiveness of antioxidants for preventing preeclampsia and other maternal and fetal complications among pregnant women with low, moderate or high risk of preeclampsia.

Methods We searched Medline, Embase, Cochrane Central, mRCT, CRD, ISI Web of Science, Lilacs, Scielo and Scopus databases, without language restriction or limits on date of publication. Randomised controlled clinical trials evaluating the use of antioxidants vs placebo or a group without antioxidants were considered eligible.
Results A total of 1120 articles were located, and 16 randomised clinical trials were analysed (20808 women). A meta-analysis did not show any statistically significant difference between women who received an antioxidant (vitamin C, vitamin E, lycopene, selenium, red palm oil) and women who received placebo, for the outcomes of preeclampsia ( $R R=0.92 ; 95 \%$ CI 0.80 to 1.06 ), severe preeclampsia $(\mathrm{RR}=1.03$; $95 \% \mathrm{CI} 0.87$ to 1.22$)$, preterm birth $(\mathrm{RR}=1.03$; $95 \% \mathrm{CI} 0.95$ to 1.11$)$, small-for-gestational-age infants $(\mathrm{RR}=0.95 ; 95 \% \mathrm{CI} 0.86$ to 1.05$)$ and any baby death $(\mathrm{RR}=1.02 ; 95 \%$ CI 0.87 to 1.20). Side-effects (abdominal pain, itching, eczema, vomiting, diarrhoea, headache, constipation, malaise, decreased vision, skin rash and chest pain) occurred more frequently among the women who took antioxidants than among those who took placebo $(\mathrm{RR}=1.58$; $95 \%$ CI 1.11 to 2.24$)$.

Conclusion The evidence does not support the use of antioxidants during pregnancy. Not only are its benefits unclear, but also adverse effects occurred more frequently with its use.

Funding MS/SCTIE/DECIT, via CNPq (Edital 67/2009).

\section{P1-282 SUBLINGUAL MISOPROSTOL FOR PREVENTING POSTPARTUM HAEMORRHAGE: A SYSTEMATIC REVIEW}

doi:10.1136/jech.2011.142976e.74

${ }^{1} \mathrm{R}$ Hoefler, ${ }^{1} \mathrm{M}$ Silva, ${ }^{1} \mathrm{~T}$ Galvao, ${ }^{1} \mathrm{~A}$ Zaconeta, ${ }^{1} \mathrm{M}$ Pereira. ${ }^{*}$ University of Brasilia, Brasilia, Distrito Federal, Brazil,; ${ }^{2}$ Centro Brasileiro de Informação Sobre Medicamentos, Brasilia, Distrito Federal, Brazil, ${ }^{3}$ Ministry of Health of Brazil, Department of Science and Technology, Brasilia, Distrito Federal, Brazil

Objective To assess the efficacy and safety of sublingual misoprostol for preventing postpartum haemorrhage.

Method MEDLINE, Embase, CRD, CENTRAL, mRCT, LILACS, SciELO, ProQuest and ISI Web of Knowledge databases were searched. There were no language, accessibility, or publication date restrictions. Randomised clinical trials of sublingual misoprostol in comparison with placebo or other uterotonics were eligible. The primary outcome was postpartum haemorrhage (blood loss $\geq 500 \mathrm{ml}$ ). Other outcomes were considered (see below). The heterogeneity was evaluated and, when possible, the data grouped into a meta-analysis using a random-effects model.

Results Of 682 references identified, only 15 were included in the analysis (5109 patients). Most of the studies were of low methodologic quality. Sublingual misoprostol, at any dose, was not more effective for reducing postpartum haemorrhage in comparison with standard treatment. Sublingual misoprostol, however, is effective for reducing haemorrhage greater than $1000 \mathrm{ml}$ [600 mcg vs placebo; $\mathrm{RR}=0.66(95 \% \mathrm{CI} 0.45$ to 0.98$)]$ as well as the use of additional uterotonics [ $600 \mathrm{mcg}$ vs methylergometrine; $\mathrm{RR}=0.04$ (0.00 to $0.72)]$ and the duration of the third stage of delivery $[50 \mathrm{mcg}$ vs methylergometrine; $\mathrm{MD}=4.16 \mathrm{~min}(2.20 ; 6.12)$ or $600 \mathrm{mcg}$ vs methylergometrine; $R R=0.04$ ( 0.00 to 0.72 )]. The drug presented a worse safety profile, causing tremors and fever, especially at higher doses.

Conclusion Sublingual misoprostol aimed at preventing postpartum haemorrhage presents no benefits compared with the standard uterotonics. Its use should be restricted to clinics with adequate medical support, as a complement to other uterotonics.

Funding MCT/CT-Saúde, MS/SCTIE/DECIT. 